\title{
Tight-binding description of Landau levels of graphite in tilted magnetic fields
}

\author{
Nataliya A. Goncharuk and Ludvík Smrčka \\ Institute of Physics, Academy of Science of the Czech Republic, v.v.i., \\ Cukrovarnická 10, 16253 Prague 6, Czech Republic \\ E-mail: gonchar@fzu.cz,smrcka@fzu.cz
}

\begin{abstract}
The electronic structure of Bernal-stacked graphite subject to a tilted magnetic field is studied theoretically. The minimal nearest-neighbor tight-binding model with the Peierls substitution is employed to describe the structure of Landau levels. We show that while the orbital effect of the in-plane component of the magnetic field is negligible for massive Dirac fermions in the vicinity of a the $K$ point of the graphite Brillouin zone, at the $H$ point it leads to the experimentally observable splitting of Landau levels, which grows approximately linearly with the in-plane field intensity.
\end{abstract}

PACS numbers: 71.20.-b, 71.70.Di

Keywords: graphite electronic structure, tilted magnetic field, splitting of Landau levels. 


\section{Introduction}

The recent attention paid to graphene monolayers has been motivated by their unusual two-dimensional (2D) Dirac energy spectrum of electrons. In Bernal-stacked graphene multilayer composed of weakly coupled graphene sheets, the interlayer interaction converts the 2D electron energy spectrum of graphene into the three-dimensional (3D) spectrum of graphite. The electronic structure of 3D graphite subject to magnetic fields perpendicular to $x-y$ planes of graphene layers were extensively studied a long time ago, see, e.g., Refs. [1, 2, 3, 4, [5, 6].

The application of the tilted magnetic field $\vec{B}=\left(0, B_{y}, B_{z}\right)$ is a standard method used to distinguish between 2D and 3D electron systems, as in 3D systems the orbital effect of the in-plane magnetic field component should be observable. We will study this problem theoretically using a simple tight-binding quantum mechanical model of the graphite electron structure.

Various approaches were employed previously to study the influence of the tilted magnetic fields.

The Fermi surfaces of metal single crystals were investigated by measurements of the de Haas-van Alphen effect in tilted magnetic fields. The interpretation of experiments relies on the quasiclassical Onsager-Lifshitz quantization rule [7, 8], the Fermi surface is reconstructed from the periods of magneto-oscillations which are proportional to angular-dependent extremal-cross-sections perpendicular to the direction of the tilted magnetic field.

In semiconductor superlattices the quasiclassical interpretation of data measured in tilted magnetic fields fails, as reported in Refs. [9, 10, 11, 12, 13, 14. In these papers the observed quantum effects are attributed to the shift of centers of $k$-space orbits in neighboring quantum wells by $|e| B_{y} / d$ in the $k_{x}$-direction, where $d$ is the distance between quantum wells. In real space this means that the in-plane magnetic-field length $\ell_{y}=\sqrt{\hbar /|e| B_{y}}$ should become comparable with $d$ to reach the visible effect [15].

Besides semiconductor superlattices, other layered materials with much shorter interlayer distances were also investigated in tilted magnetic fields. Different versions of angular magnetoresistance oscillations (AMRO) were studied both experimentally and theoretically in low-dimensional quasi-2D and quasi-one-dimensional organic conductors (see, e.g.,Refs. [16, 17, 18, 19, 20, 21, 22, 23, 24] and references therein), and also in intercalated graphite [25, 26]. On the theory side, the high Landau level (LL) filing factors and weak interlayer interaction were considered in iterpretation the data.

In pristine graphite this problem has been touched on by two recent theoretical articles.

The graphene multilayer energy spectrum in magnetic fields parallel to the layers was described quantum-mechanically in Ref. [27], as the standard theory of AMRO in tilted magnetic fields was not applicable due to the relatively strong interlayer interaction (in comparison with the intercalated graphite) between graphene sheets.

The LLs in the bilayer graphene in magnetic fields of arbitrary orientations were 
calculated analytically in Ref. [28].

Both papers conclude that a very strong in-plane field component is necessary to induce an observable effect on the electronic structure. Indeed, to reach $\ell_{y}$ comparable with the distance between graphene layers in graphite, the magnetic field $B_{y}=5865 \mathrm{~T}$ would be necessary.

In this paper we make use of the specific features of the LL structure in two nonequivalent neighboring graphene sheets in graphite, and show that at the $H$ point of the graphite hexagonal Brillouin zone the application of the tilted magnetic field leads to experimentally observable splitting of LLs of the order of several meV.

\section{Model}

Bulk graphite is composed of periodically repeated graphene bilayers formed by two nonequivalent Bernal-stacked graphene sheets, as shown in Fig. 1. There are two sublattices, $A$ and $B$, on each sheet and, therefore, four atoms in a unit cell. The distance between the nearest atoms $A$ and $B$ in a single layer is $1.42 \AA$, the interlayer distance between nearest atoms $A$ is $d=3.35 \AA$.

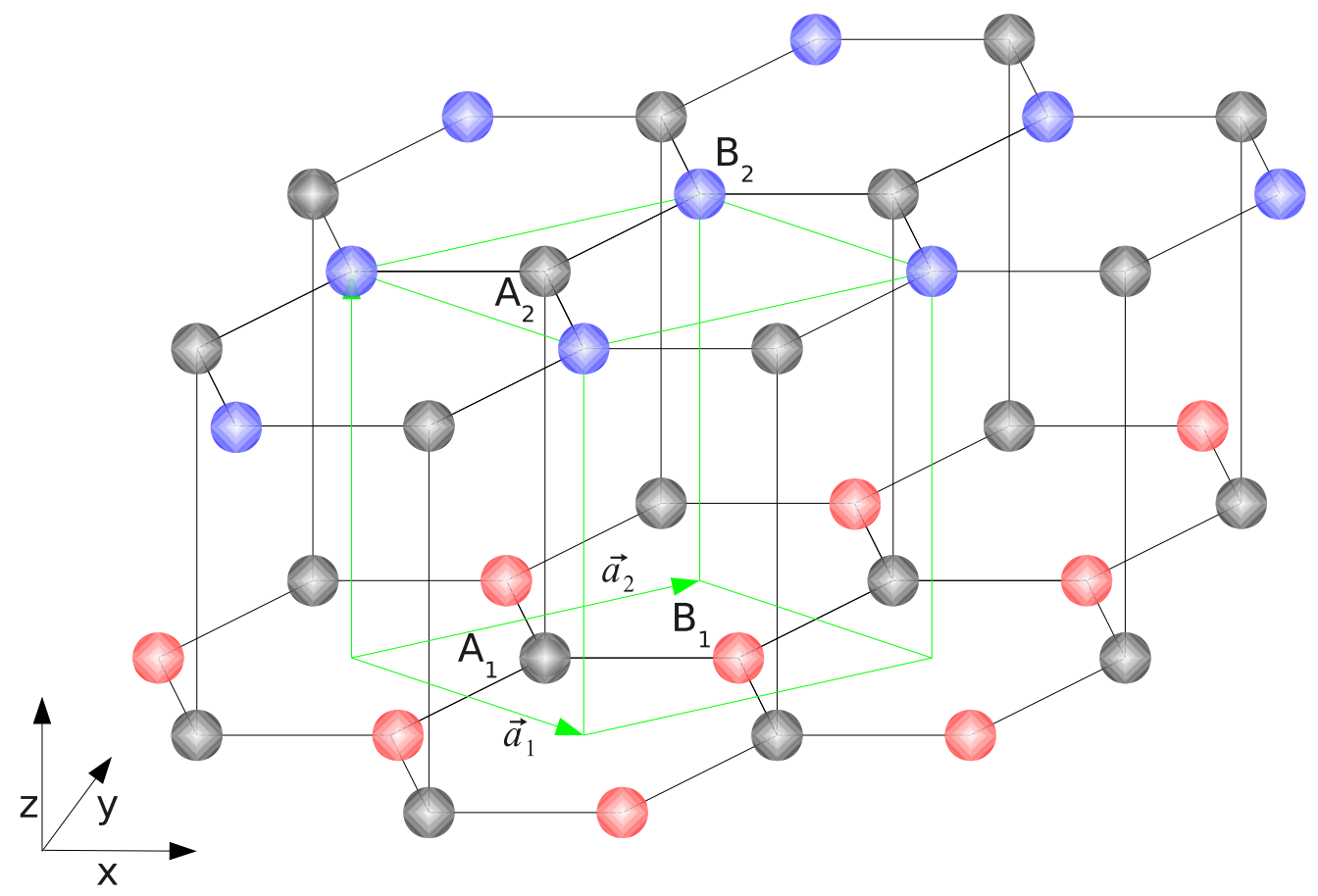

Figure 1. (Color online) The lattice structure of graphite. The unit cell is a green parallelepiped.

To describe the graphite band structure, we employ the minimal nearest-neighbor tight-binding model, introduced by Koshino et al. in Ref. [29]. This model is reduced Slonczewski, Weiss and McClure (SWM) model. The tight-binding Hamiltonian $\mathcal{H}$ includes only two instead of seven tight-binding parameters, the intralayer interaction 
$\gamma_{0}=3.16 \mathrm{eV}$ between the nearest atoms $A$ and $B$ in the plane, and the interlayer interaction $t=0.39 \mathrm{eV}$ between the nearest atoms $A$ out of plane.

While the reduced SWM model is not appropriate, e.g., for a Fermi surface description, this model has been successfully applied in the theoretical papers [27, 28], and used to describe recent magneto-optical measurements on graphite in Refs. 31, 32, 30, 33, 34. It has been shown in Refs. 30, 33, 34 that the transitions between Landau levels originating from the $K$ and $H$ points of the graphite Brillouine zone can be understood within a simple picture of an effective bilayer with a coupling strength enhanced twice in comparison to a true graphene bilayer, $2 t$, (which is definitely the effect of a superlattice) and an effective graphene monolayer. These solid arguments are in agreement with the theoretical model based on the reduced SWM model we develop in our manuscript.

In this model the wave functions are expressed via four orthogonal components $\psi_{j}^{A}$, $\psi_{j}^{B}, \psi_{j+1}^{A}, \psi_{j+1}^{B}$, which are, in zero magnetic field, Bloch sums of atomic wave functions over the lattice sites of sublattices $A$ and $B$ in individual layers $j$.

The continuum approximation is used in the vicinity of the $H-K-H$ axis of the graphite hexagonal Brillouin zone, for small $\vec{k}=\left(k_{x}, k_{y}\right)$ measured from the axis. Then the electron wave length is larger than the distance between atoms, and the non-zero matrix elements of $\mathcal{H}$ can be written as

$$
\begin{aligned}
& \mathcal{H}^{A B}=\hbar v_{F}\left(k_{x}+i k_{y}\right), \\
& \mathcal{H}^{B A}=\hbar v_{F}\left(k_{x}-i k_{y}\right), \\
& \mathcal{H}^{A A}=t .
\end{aligned}
$$

The Fermi velocity, $v_{F}$, is defined by $\hbar v_{F}=\sqrt{3} a \gamma_{0} / 2$, and will be used as an intralayer parameter instead of $\gamma_{0}$ in the subsequent consideration.

The effect of the arbitrary oriented magnetic field, $\vec{B}=\left(0, B_{y}, B_{z}\right)$, can be conveniently introduced into the zero-field Hamiltonian by the Peierls substitution. If we choose the vector potential in the Landau form $\vec{A}=\left(B_{y} z-B_{z} y, 0,0\right)$, the substitution will read

$$
\hbar k_{x} \rightarrow \hbar k_{x}-|e| B_{z} y+|e| B_{y} j d,
$$

where an integer number $j$ indicates the graphite layer number. Consequently, the matrix elements, $\mathcal{H}^{A B}$ and $\mathcal{H}^{B A}$, become layer dependent in a tilted magnetic field,

$$
\begin{aligned}
& \mathcal{H}_{j}^{A B}=v_{F}\left(k_{x}-|e| B_{z} y+|e| B_{y} j d+i k_{y}\right)=v_{F} \Pi_{j}, \\
& \mathcal{H}_{j}^{B A}=v_{F}\left(k_{x}-|e| B_{z} y+|e| B_{y} j d-i k_{y}\right)=v_{F} \Pi_{j}^{*} .
\end{aligned}
$$

Making use of the above approximations, the Schrödinger equation involving all layers $j$, leads to the following system of equations

$$
\begin{aligned}
& v_{F} \Pi_{j}^{*} \psi_{j}^{B}-E \psi_{j}^{A}+t \psi_{j-1}^{A}+t \psi_{j+1}^{A}=0, \\
& -E \psi_{j}^{B}+v_{F} \Pi_{j}^{*} \psi_{j}^{A}=0, \\
& v_{F} \Pi_{j+1}^{*} \psi_{j+1}^{B}-E \psi_{j+1}^{A}+t \psi_{j}^{A}+t \psi_{j+2}^{A}=0, \\
& -E \psi_{j+1}^{B}+v_{F} \Pi_{j+1} \psi_{j+1}^{A}=0 .
\end{aligned}
$$


Note that the structure of $\mathcal{H}$ allows to express the function $\psi_{j}^{B}$ via the function $\psi_{j}^{A}$ from the same layer, and we are thus left with two interlayer equations for $\psi_{j}^{A}$ and $\psi_{j+1}^{A}$

$$
\begin{aligned}
& \left(v_{F}^{2} \Pi_{j} \Pi_{j}^{*}-E^{2}\right) \psi_{j}^{A}+t E\left(\psi_{j-1}^{A}+\psi_{j+1}^{A}\right)=0, \\
& \left(v_{F}^{2} \Pi_{j+1}^{*} \Pi_{j+1}-E^{2}\right) \psi_{j+1}^{A}+t E\left(\psi_{j}^{A}+\psi_{j+2}^{A}\right)=0 .
\end{aligned}
$$

\section{Zero-field case}

It follows from the condition of periodicity in the $z$-direction that $\psi_{j+1}^{A}$ and $\psi_{j}^{A}$ can be written as

$$
\psi_{j+1}^{A}=e^{i k_{z} d(j+1)} \phi_{1}^{A}, \quad \psi_{j}^{A}=e^{i k_{z} d j} \phi_{2}^{A},
$$

where $\phi_{1}^{A}$ and $\phi_{2}^{A}$ denote the 2D wave functions in two non-equivalent layers of the graphite unite cell, and $k_{z}$ is restricted to the first Brillouin zone, $-\pi / 2<k_{z} d<\pi / 2$.

For $\vec{B}=0$ Eqs. (11, 12) are transformed to

$$
\begin{aligned}
& \left(\hbar^{2} v_{F}^{2} k^{2}-E^{2}\right) \phi_{2}^{A}+2 t E \cos \left(k_{z} d\right) \phi_{1}^{A}=0, \\
& \left(\hbar^{2} v_{F}^{2} k^{2}-E^{2}\right) \phi_{1}^{A}+2 t E \cos \left(k_{z} d\right) \phi_{2}^{A}=0,
\end{aligned}
$$

and from here the four eigenvalues are obtained

$$
E^{ \pm, \pm}= \pm \mathcal{T} \pm \sqrt{\mathcal{T}^{2}+\hbar^{2} v_{F}^{2} k^{2}}
$$

where

$$
\mathcal{T}=t \cos \left(k_{z} d\right)
$$

denotes the $k_{z}$-dependent coupling of two graphene sheets.

\section{Perpendicular magnetic field}

In the perpendicular magnetic field, $B_{y}=0$, the system remains periodic in the $z$ direction, and Eq. (13) is still valid. To find a proper form of $\mathcal{H}^{A B}$ and $\mathcal{H}^{B A}$ we introduce the perpendicular magnetic field length, $\ell_{z}^{2}=\hbar /\left(|e| B_{z}\right)$, the centre of a cyclotron orbit, $y_{0}=\ell_{z}^{2} k_{x}$, the dimensionless variable, $\eta=\left(y-y_{0}\right) / \ell_{z}$, and the perpendicular-magneticfield-dependent parameter, $\mathcal{B}=2 \hbar|e| B_{z} v_{F}^{2}$. Then, in our notation,

$$
\begin{aligned}
& \mathcal{H}^{A B}=v_{F} \Pi=-\sqrt{\mathcal{B}} a^{\dagger}=-\sqrt{\frac{\mathcal{B}}{2}}\left(-\frac{\partial}{\partial \eta}+\eta\right), \\
& \mathcal{H}^{B A}=v_{F} \Pi^{*}=-\sqrt{\mathcal{B}} a=-\sqrt{\frac{\mathcal{B}}{2}}\left(\frac{\partial}{\partial \eta}+\eta\right),
\end{aligned}
$$

$a^{\dagger}$ and $a$ being raising and lowering operators, respectively.

With help of these expressions, Eqs. (11) and (12) can be written as

$$
\begin{aligned}
& {\left[\frac{\mathcal{B}}{2}\left(-\frac{\partial^{2}}{\partial \eta^{2}}+\eta^{2}+1\right)-E^{2}\right] \phi_{2}^{A}+2 \mathcal{T} E \phi_{1}^{A}=0,} \\
& {\left[\frac{\mathcal{B}}{2}\left(-\frac{\partial^{2}}{\partial \eta^{2}}+\eta^{2}-1\right)-E^{2}\right] \phi_{1}^{A}+2 \mathcal{T} E \phi_{2}^{A}=0 .}
\end{aligned}
$$


It is obvious from these equations that $\phi_{1}^{A}$ and $\phi_{2}^{A}$ are closely related to the eigenfunctions of the harmonic oscillator, $\varphi_{n}(\eta)$. Assuming

$$
\phi_{1}^{A}=\frac{1}{L_{x}} e^{i k_{x} x} \sum_{n^{\prime}=0}^{\infty} A_{1, n^{\prime}} \varphi_{n^{\prime}}(\kappa), \quad \phi_{2}^{A}=\frac{1}{L_{x}} e^{i k_{x} x} \sum_{n^{\prime}=0}^{\infty} A_{2, n^{\prime}} \varphi_{n^{\prime}}(\kappa),
$$

and having in mind that

$$
\left(-\frac{\partial^{2}}{\partial \eta^{2}}+\eta^{2}\right) \varphi_{n}(\eta)=(2 n+1) \varphi_{n}(\eta)
$$

we get

$$
\begin{aligned}
& {\left[\mathcal{B}(n+1)-E^{2}\right] A_{2, n}+2 E \sum_{n^{\prime}=0}^{\infty} \mathcal{T}_{n, n^{\prime}} A_{1, n^{\prime}}=0,} \\
& {\left[\mathcal{B} n-E^{2}\right] A_{1, n}+2 E \sum_{n^{\prime}=0}^{\infty} \mathcal{T}_{n, n^{\prime}} A_{2, n^{\prime}}=0,}
\end{aligned}
$$

where $T_{n, n^{\prime}}$ is defined by

$$
\mathcal{T}_{n, n^{\prime}}=\mathcal{T} \int_{-\infty}^{+\infty} \varphi_{n}(\eta) \varphi_{n^{\prime}}(\eta) d \eta=\mathcal{T} \delta_{n, n^{\prime}}
$$

It follows from (26) that only the LLs with the same quantum numbers $n$ (but with the different energies) are coupled and we arrive to

$$
\begin{aligned}
& {\left[\mathcal{B}(n+1)-E^{2}\right] A_{2, n}+2 \mathcal{T}_{n, n} E A_{1, n}=0,} \\
& \left(\mathcal{B} n-E^{2}\right) A_{1, n}+2 \mathcal{T}_{n, n} E A_{2, n}=0
\end{aligned}
$$

Solving Eqs. (27, 28) yields the eigenenergies

$$
E_{n}^{ \pm, \pm}= \pm \sqrt{2 \mathcal{T}_{n, n}^{2}+\mathcal{B}\left(n+\frac{1}{2}\right) \pm \sqrt{4 \mathcal{T}_{n, n}^{4}+4 \mathcal{T}_{n, n}^{2} \mathcal{B}\left(n+\frac{1}{2}\right)+\frac{\mathcal{B}^{2}}{4}}}
$$

which are presented in Fig. 2.

As the densities of states of the above Landau subbands have singularities at the points $K$ and $H, k_{z} d=0$ and $k_{z} d=\pi / 2$, respectively, we concentrate on the field dependence of levels corresponding to these points.

At the $K$ point the eigenenergies are given by

$$
E_{n}^{ \pm, \pm}= \pm \sqrt{2 t^{2}+\mathcal{B}\left(n+\frac{1}{2}\right) \pm \sqrt{4 t^{4}+4 t^{2} \mathcal{B}\left(n+\frac{1}{2}\right)+\frac{\mathcal{B}^{2}}{4}}}
$$

i.e., the subbands are equivalent to those of a graphene bilayer with the coupling constant doubled: $2 t$ instead of $t$.

At the $H$ point the eigenenergies read

$$
E_{n}^{ \pm, \pm}= \pm \sqrt{\mathcal{B}\left(n+\frac{1}{2}\right) \mp \frac{\mathcal{B}}{2}}
$$

Due to the effectively vanishing inter-layer coupling the spectrum corresponds to the Dirac fermions. The coefficients $A_{1, n}$ and $A_{2, n}$ in Eqs. (27, 28) are equal to 1 and this 


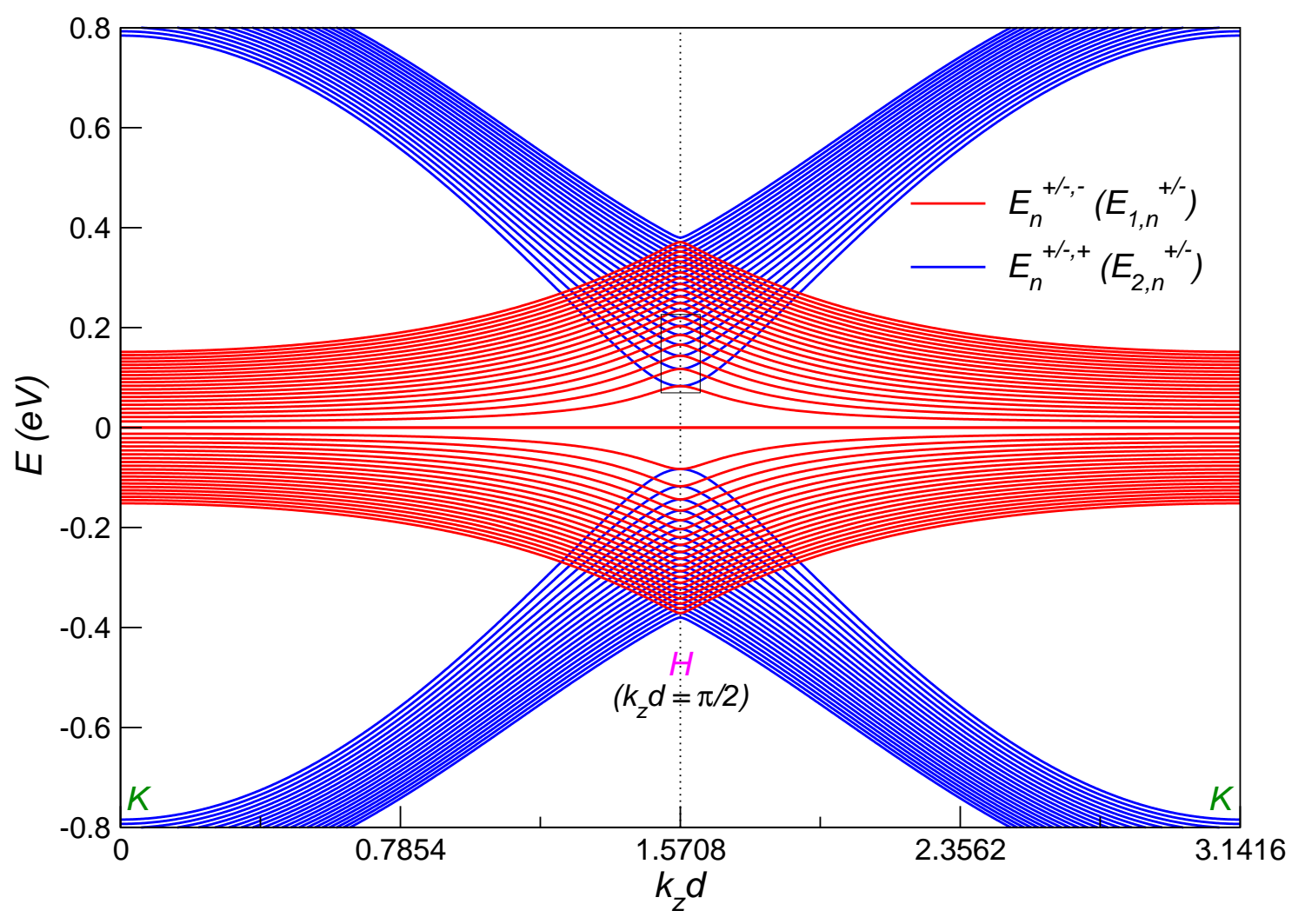

Figure 2. (Color online) Landau subbands of graphite, $E_{n}^{ \pm,-}$and $E_{n}^{ \pm,+}$(denoted as $E_{1, n}^{ \pm}$and $E_{2, n}^{ \pm}$at the $H$ point) subject to perpendicular magnetic field $B_{z}=5 \mathrm{~T}$, as a function of $k_{z}$ along the K-H-K pass of the Brillouin zone. The $k_{z}$ dependence of LLs in the vicinity of the $H$ point restricted by the rectangular box will be compared with those subject to the tilted magnetic field, as shown in Fig. 6 .

implies that the corresponding wave functions are localized either in the layer 1 or in the layer 2. To emphasize it, we will write the eigeneneries as

$$
\begin{aligned}
& E_{1, n}^{ \pm}=E_{n}^{ \pm,-}= \pm \sqrt{\mathcal{B} n}, \\
& E_{2, n}^{ \pm}=E_{n}^{ \pm,+}= \pm \sqrt{\mathcal{B}(n+1)} .
\end{aligned}
$$

The energy spectrum of LLs at the $H$ point is presented in Fig. 3 .

We also denote the wave functions $\phi_{1(2)}^{A}$ and $\phi_{1(2)}^{B}$ as $|n\rangle_{1(2)}^{A}$ and $|n\rangle_{1(2)}^{B}$ to stress that they are the envelope wave functions of atomic wave functions $A$ and $B$ in the layers 1 and 2, as shown in TABLE 1.

Let us mention that $E_{2, n}^{ \pm}=E_{1, n+1}^{ \pm}$, i.e., we are left with pairs of degenerated LLs with different quantum numbers $n$ but the same eigenenergies, with the wave functions localized in two different layers.

\section{Tilted magnetic field}

While the previous two paragraphs summarized the already published theories devoted to $\vec{B}=0$ and $\vec{B}=\left(0,0, B_{z}\right)$, here we present new results for $\vec{B}=\left(0, B_{y}, B_{z}\right)$. 
Tight-binding description of Landau levels of graphite in tilted magnetic fields



Figure 3. (Color online) LLs of graphite at the $H$ point of the Brillouin zone, $E_{1, n}^{ \pm}$ and $E_{2, n}^{ \pm}$, in the perpendicular magnetic field, $B_{z}$.

Table 1. Energies and wave functions at the graphite $H$ point in perpendicular magnetic fields

\begin{tabular}{|c|c|}
\hline Energy & $\left\{\phi_{1}^{A}, \phi_{1}^{B}, \phi_{2}^{A}, \phi_{2}^{B}\right\}$ \\
\hline$E_{1}^{+}=\sqrt{\mathcal{B} n}$ & $\left\{|n\rangle_{1}^{A},-|n-1\rangle_{1}^{B}, 0,0\right\}$ \\
\hline$E_{1}^{-}=-\sqrt{\mathcal{B} n}$ & $\left\{|n\rangle_{1}^{A},|n-1\rangle_{1}^{B}, 0,0\right\}$ \\
\hline$E_{2}^{+}=\sqrt{\mathcal{B}(n+1)}$ & $\left\{0,0,|n\rangle_{2}^{A},-|n+1\rangle_{2}^{B}\right\}$ \\
\hline$E_{2}^{-}=-\sqrt{\mathcal{B}(n+1)}$ & $\left\{0,0,|n\rangle_{2}^{A},|n+1\rangle_{2}^{B}\right\}$ \\
\hline
\end{tabular}




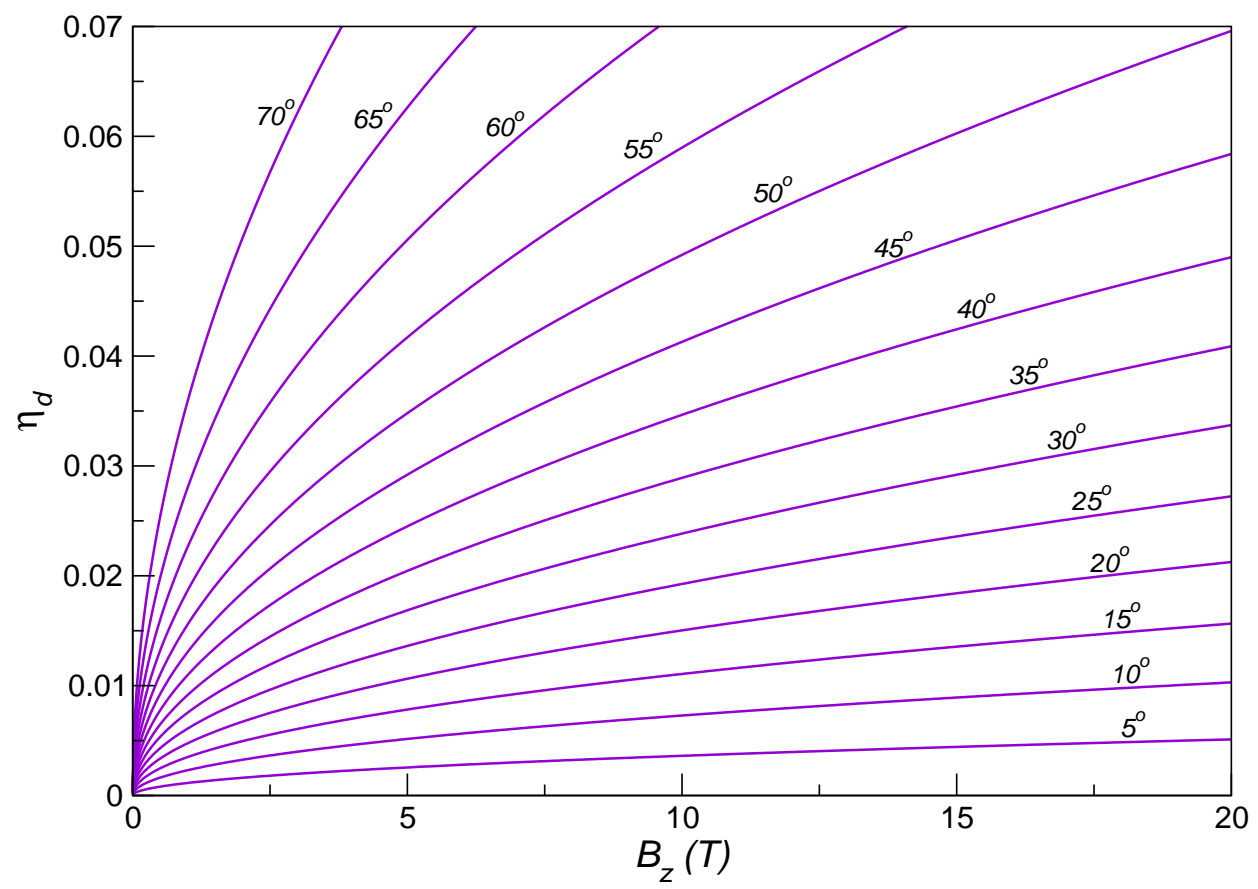

Figure 4. (Color online) The dimensionless parameter $\eta_{d}$ as a function of the perpendicular component of the magnetic field, $B_{z}$, calculated for various tilt angles of the magnetic field $\vec{B}$.

In tilted magnetic fields the off-diagonal matrix elements, $\mathcal{H}_{j}^{A B}$ and $\mathcal{H}_{j}^{B A}$, given by Eqs. (5, 6) remain layer dependent and take the form

$$
\begin{aligned}
& \mathcal{H}_{j}^{A B}=v_{F} \Pi_{j}=-\sqrt{\frac{\mathcal{B}}{2}}\left(-\frac{\partial}{\partial \eta}+\eta-j \eta_{d}\right), \\
& \mathcal{H}_{j}^{B A}=v_{F} \Pi_{j}^{*}=-\sqrt{\frac{\mathcal{B}}{2}}\left(\frac{\partial}{\partial \eta}+\eta-j \eta_{d}\right),
\end{aligned}
$$

where the small dimensionless parameter

$$
\eta_{d}=\frac{B_{y}}{B_{z}} \frac{d}{\ell_{z}}
$$

means the shift of the cyclotron orbit center in the $j$-layer due to the in-plane component of the magnetic field, $B_{y}$. Fig. 团illustrates the $B_{z}$ dependence of $\eta_{d}$ for various tilt angles of the magnetic field.

Note that graphite subject to tilted magnetic fields is no longer periodic in the $z$ direction, but becomes periodic in the direction of the tilted magnetic field. To take into account the shift of the cyclotron orbits, we apply the approach developed in Ref. 35] for semiconductor superlattices. Accordingly, the Eqs. (11) and (12) are modified to

$$
\begin{aligned}
& {\left[\frac{\mathcal{B}}{2}\left(-\frac{\partial^{2}}{\partial \eta^{2}}+\left(\eta-j \eta_{d}\right)^{2}+1\right)-E^{2}\right] \psi_{j}^{A}+t E\left(\psi_{j-1}^{A}+\psi_{j+1}^{A}\right)=0,} \\
& {\left[\frac{\mathcal{B}}{2}\left(-\frac{\partial^{2}}{\partial \eta^{2}}+\left(\eta-(j-1) \eta_{d}\right)^{2}-1\right)-E^{2}\right] \psi_{j+1}^{A}+} \\
& +t E\left(\psi_{j}^{A}+\psi_{j+2}^{A}\right)=0 .
\end{aligned}
$$


The new periodicity implies that $\psi_{j}^{A}$ and $\psi_{j+1}^{A}$ can be written as

$$
\psi_{j}^{A}=e^{i k_{z} d j} \phi_{1}^{A}\left(\eta+j \eta_{d}\right), \quad \psi_{j+1}^{A}=e^{i k_{z} d(j+1)} \phi_{2}^{A}\left[\eta+(j+1) \eta_{d}\right],
$$

where again $-\pi / 2 \leq k_{z} d \leq \pi / 2$. Here the wave functions $\phi_{1}^{A}\left(\eta+j \eta_{d}\right)$ and $\phi_{2}^{A}\left(\eta+j \eta_{d}\right)$ are associated with cyclotron orbits in two layers. Introducing the shift operator by

$$
\phi\left(\eta+\eta_{d}\right)=e^{i \kappa \eta_{d}} \phi(\eta), \quad \kappa=-i \partial / \partial \eta,
$$

and employing the $\kappa$-representation, two interlayer Eqs. (37) and (38) can be given the form similar to Eqs. (20, 21) for the perpendicular magnetic field

$$
\begin{aligned}
& {\left[\frac{\mathcal{B}}{2}\left(-\frac{\partial^{2}}{\partial \kappa^{2}}+\kappa^{2}+1\right)-E^{2}\right] \phi_{2}^{A}+2 \widetilde{\mathcal{T}} E \phi_{1}^{A}=0,} \\
& {\left[\frac{\mathcal{B}}{2}\left(-\frac{\partial^{2}}{\partial \kappa^{2}}+\kappa^{2}-1\right)-E^{2}\right] \phi_{1}^{A}+2 \widetilde{\mathcal{T}} E \phi_{2}^{A}=0}
\end{aligned}
$$

but with the new coupling $\widetilde{\mathcal{T}}$ which depends, due to new periodicity, not only on $k_{z}$ but also on both components $B_{z}$ and $B_{y}$ of the arbitrary oriented magnetic field via $\eta_{d}$,

$$
\tilde{\mathcal{T}}(\kappa)=t \cos \left(\kappa \eta_{d}+k_{z} d\right) .
$$

The Eqs. (41) and (42) represent the main result of this paper and in the following we will discuss the possible methods of their solutions.

Expressing again $\phi_{1}^{A}$ and $\phi_{2}^{A}$ with a help of (24) and (25) we arrive to

$$
\begin{aligned}
& {\left[\mathcal{B}(n+1)-E^{2}\right] A_{2, n}+2 E \sum_{n^{\prime}=0}^{\infty} \widetilde{\mathcal{T}}_{n, n^{\prime}} A_{1, n^{\prime}}=0,} \\
& {\left[\mathcal{B} n-E^{2}\right] A_{1, n}+2 E \sum_{n^{\prime}=0}^{\infty} \widetilde{\mathcal{T}}_{n, n^{\prime}} A_{2, n^{\prime}}=0,}
\end{aligned}
$$

where

$$
\widetilde{\mathcal{T}}_{n, n^{\prime}}=\int_{-\infty}^{\infty} \varphi_{n}(\kappa) \widetilde{\mathcal{T}}(\kappa) \varphi_{n^{\prime}}(\kappa) d \kappa .
$$

The integrals (46) can be evaluated analytically and expressed via the generalised Laguerre polynomials (see, e.g., Ref. [36]). The Eqs. (44) and (45) define the matrix which should be diagonalized. The nonzero coupling of LLs with different $n$ allows us to conclude that the degeneracy of LLs at the $H$ point will be removed, and the LLs with different $n$ avoid to cross. Then the standard approach is to solve the secular equation numerically, the minor complication being that the matrix elements depend on the energy.

Here we prefer to obtain analytic results by the lowest order perturbation theory application and treating $\eta_{d}$ as a small parameter.

A simple trigonometric relation and a series expansion restricted to terms linear in $\eta_{d}$ imply

$$
\begin{aligned}
\tilde{\mathcal{T}}(\kappa) & =t \cos \left(k_{z} d\right) \cos \left(\kappa \eta_{d}\right)-t \sin \left(k_{z} d\right) \sin \left(\kappa \eta_{d}\right) \approx \\
& \approx t \cos \left(k_{z} d\right)-t \sin \left(k_{z} d\right) \eta_{d} \kappa \cdots,
\end{aligned}
$$


and

$$
\widetilde{\mathcal{T}}_{n, n^{\prime}}=t \cos \left(k_{z} d\right) \delta_{n, n^{\prime}}-t \eta_{d} \sin \left(k_{z} d\right) \int_{-\infty}^{\infty} \varphi_{n}(\kappa) \kappa \varphi_{n^{\prime}}(\kappa) d \kappa
$$

The integrals in Eq. (48) can be easily evaluated using the relation (see, e.g., Ref. [37])

$$
\kappa \varphi_{n}(\kappa)=\sqrt{\frac{n+1}{2}} \varphi_{n+1}(\kappa)+\sqrt{\frac{n}{2}} \varphi_{n-1}(\kappa) .
$$

Let us pay attention to the field dependence of LLs at the most interesting $K$ and $H$ points of the graphite Brillouin zone.

At the $K$ point $k_{z} d=0$, and, consequently, $\cos \left(k_{z} d\right)=1, \sin \left(k_{z} d\right)=0$. Then $\widetilde{\mathcal{T}}_{n, n^{\prime}}$ reduces to

$$
\widetilde{\mathcal{T}}_{n, n^{\prime}}=t \delta_{n, n^{\prime}}
$$

This coupling corresponds to the effective bilayer subject to the perpendicular field discussed above. Thus, we have found that corrections induced by $B_{y}$ are very small and of the order $\eta_{d}^{2}$. This is in agreement with conclusions presented in Ref. [28].

The field dependence at the $H$ point, $k_{z} d= \pm \pi / 2$, is more interesting. In perpendicular magnetic fields the coupling between layers disappears, and, as presented above, we obtained the LLs corresponding to graphene Dirac fermions, namely $E_{1, n}^{ \pm}=$ $\pm \sqrt{\mathcal{B} n}$ for the first layer, and $E_{2, n}^{ \pm}= \pm \sqrt{\mathcal{B}(n+1)}$ for the second layer, $n=0,1,2, \cdots$.

In the magnetic field of an arbitrary direction the interlayer interaction is not reduced to zero, but remains finite. The non-zero matrix elements $\widetilde{\mathcal{T}}_{n, n^{\prime}}$ can be written as

$$
\tilde{\mathcal{T}}_{n, n+1}=\tilde{\mathcal{T}}_{n+1, n}=t \eta_{d} \sqrt{\frac{n+1}{2}}, \quad \tilde{\mathcal{T}}_{n, n-1}=\tilde{\mathcal{T}}_{n-1, n}=t \eta_{d} \sqrt{\frac{n}{2}} .
$$

The small perturbation $\eta_{d}$ couples the states $|n\rangle_{2}^{A}$ with $|n+1\rangle_{1}^{A}$ and $|n-1\rangle_{1}^{A}$. Among them the states $|n\rangle_{2}^{A}$ and $|n+1\rangle_{1}^{A}$ are degenerated, i.e., they belong to the same unpertubed eigenvalues $\pm \sqrt{\mathcal{B}(n+1)}$. Consequently, at least the lowest order perturbation approach suitable to remove the degeneracy must be applied, which yields equations

$$
\begin{aligned}
& {\left[\mathcal{B}(n+1)-E^{2}\right] A_{2, n}+2 E \widetilde{\mathcal{T}}_{n, n+1} A_{1, n+1}=0,} \\
& {\left[\mathcal{B}(n+1)-E^{2}\right] A_{1, n+1}+2 E \widetilde{\mathcal{T}}_{n+1, n} A_{2, n}=0 .}
\end{aligned}
$$

The secular equation derived from Eqs. (52, 53) reads

$$
\left[\mathcal{B}(n+1)-E^{2}\right]^{2}-4 E^{2} \widetilde{\mathcal{T}}_{n, n+1}^{2}=0,
$$

and from here we get the four eigenenergies

$$
E_{n+1}^{ \pm, \pm}= \pm \widetilde{\mathcal{T}}_{n, n+1} \pm \sqrt{\mathcal{B}(n+1)+\widetilde{\mathcal{T}}_{n, n+1}^{2}}, \quad n=0,1,2, \cdots
$$

The eigenenergies, $E_{0}^{\mp}$ and $E_{0}^{ \pm}$, steming from $E_{1,0}^{ \pm}$, remain the same as in the perpendicular magnetic field. In that case the degeneracy is not removed. 


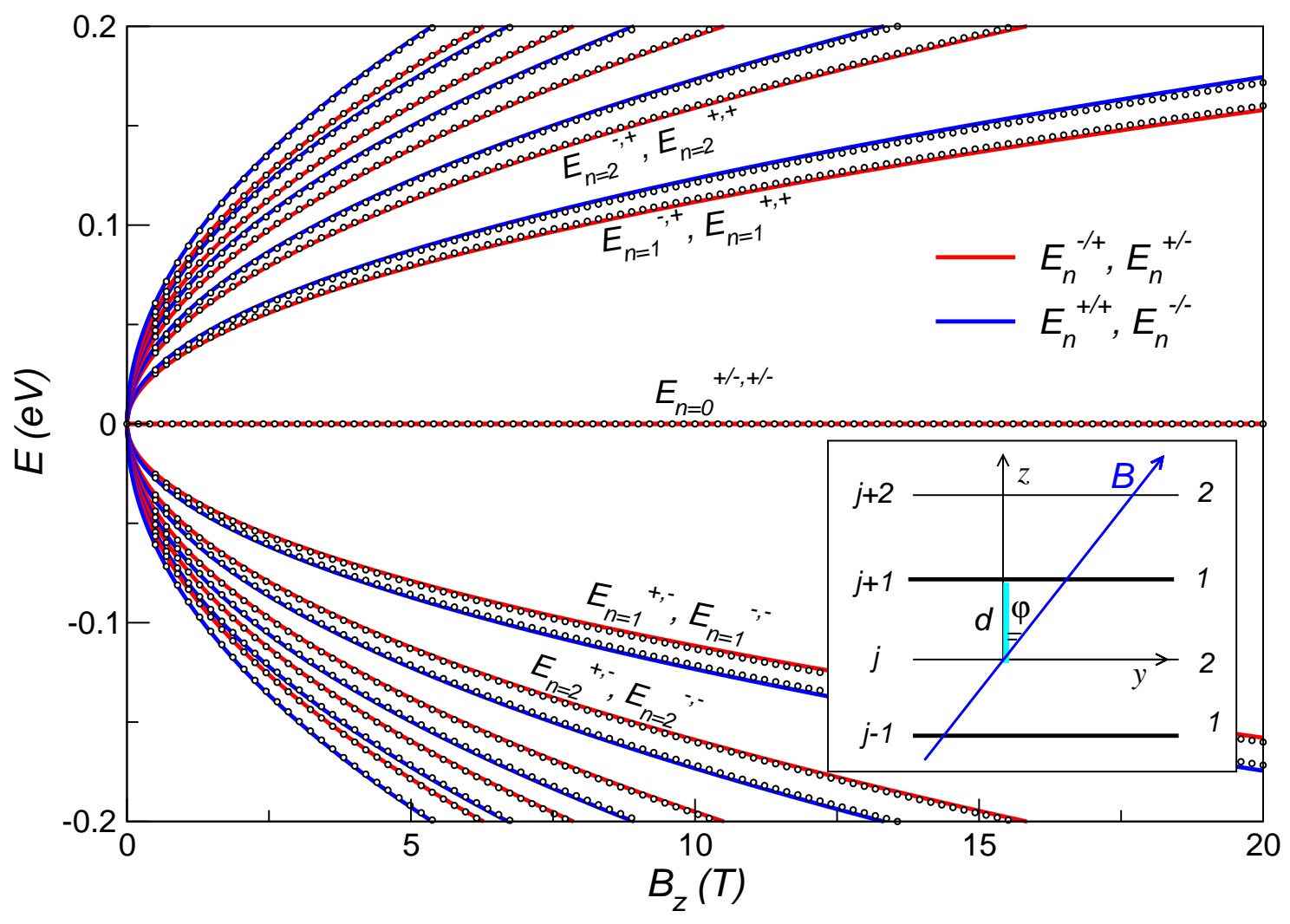

Figure 5. (Color online) Splitted by tilting the magnetic field with $\varphi=20^{\circ}$ LLs of graphite at the $H$ point, $E_{n}^{ \pm, \pm}$, as a function of the perpendicular component of the magnetic field, $B_{z}$. The dotted lines correspond to results of numerical calculations.

Lifting of LL degeneracy by the tilted magnetic field in LLs with $n>0$ is shown in Fig. 5. The LL splitting is of the order of several meV, and it grows with the tilt angle, i.e., with the in-plane magnetic field component, $B_{y}$.

The corresponding eigenfunctions calculated with the same level of accuracy are presented in TABLE 2. They are mixed from wave functions of both layers with an equal weight.

To test the accuracy of the above approximations we have calculated the eigenvalues numerically with the larger basis $|n\rangle_{1}^{A},|n+1\rangle_{1}^{A},|n+2\rangle_{1}^{A},|n\rangle_{2}^{A},|n+1\rangle_{2}^{A},|n+2\rangle_{2}^{A}$ instead of the minimal one $|n+1\rangle_{1}^{A},|n\rangle_{2}^{A}$. At the $H$ point we have found only negligible quantitative corrections to the results obtained analytically, as presented in Fig. 5. Similarly, including the higher order expansion in $\eta_{d}$ does not influence the results for the chosen range of angles and magnetic fields.

The above basis allows to calculate also the $k_{z}$ dependence in the vicinity of the $H$ point defined roughly by the rectangle in Fig. 2. The most interesting feature is the development of additional local extrema near the $H$ point, which are more pronounced for LLs with higher $n$. The same is true for minigaps open at the crossing points of LLs, as mentioned in the previous paragraphs. The results are shown in Fig. 6.

In general, our approach must fail for magnetic fields close to the in-plane 
Table 2. Energies and wave functions in tilted magnetic fields at the graphite $H$ point

\begin{tabular}{|c|c|}
\hline Energy & $\left\{\phi_{1}^{A}, \phi_{1}^{B}, \quad \phi_{2}^{A}, \quad \phi_{2}^{B}\right\}$ \\
\hline$E_{n}^{\mp}$ & $\left\{|n\rangle_{1}^{A},-|n-1\rangle_{1}^{B},-|n+1\rangle_{2}^{A},|n+2\rangle_{2}^{B}\right\}$ \\
\hline$E_{n}^{--}$ & $\left\{|n\rangle_{1}^{A},|n-1\rangle_{1}^{B},-|n+1\rangle_{2}^{A},-|n+2\rangle_{2}^{B}\right\}$ \\
\hline$E_{n}^{++}$ & $\left\{|n+1\rangle_{1}^{A},-|n\rangle_{1}^{B},|n\rangle_{2}^{A},-|n+1\rangle_{2}^{B}\right\}$ \\
\hline$E_{n}^{ \pm}$ & $\left\{|n+1\rangle_{1}^{A},|n\rangle_{1}^{B},|n\rangle_{2}^{A},|n+1\rangle_{2}^{B}\right\}$ \\
\hline
\end{tabular}

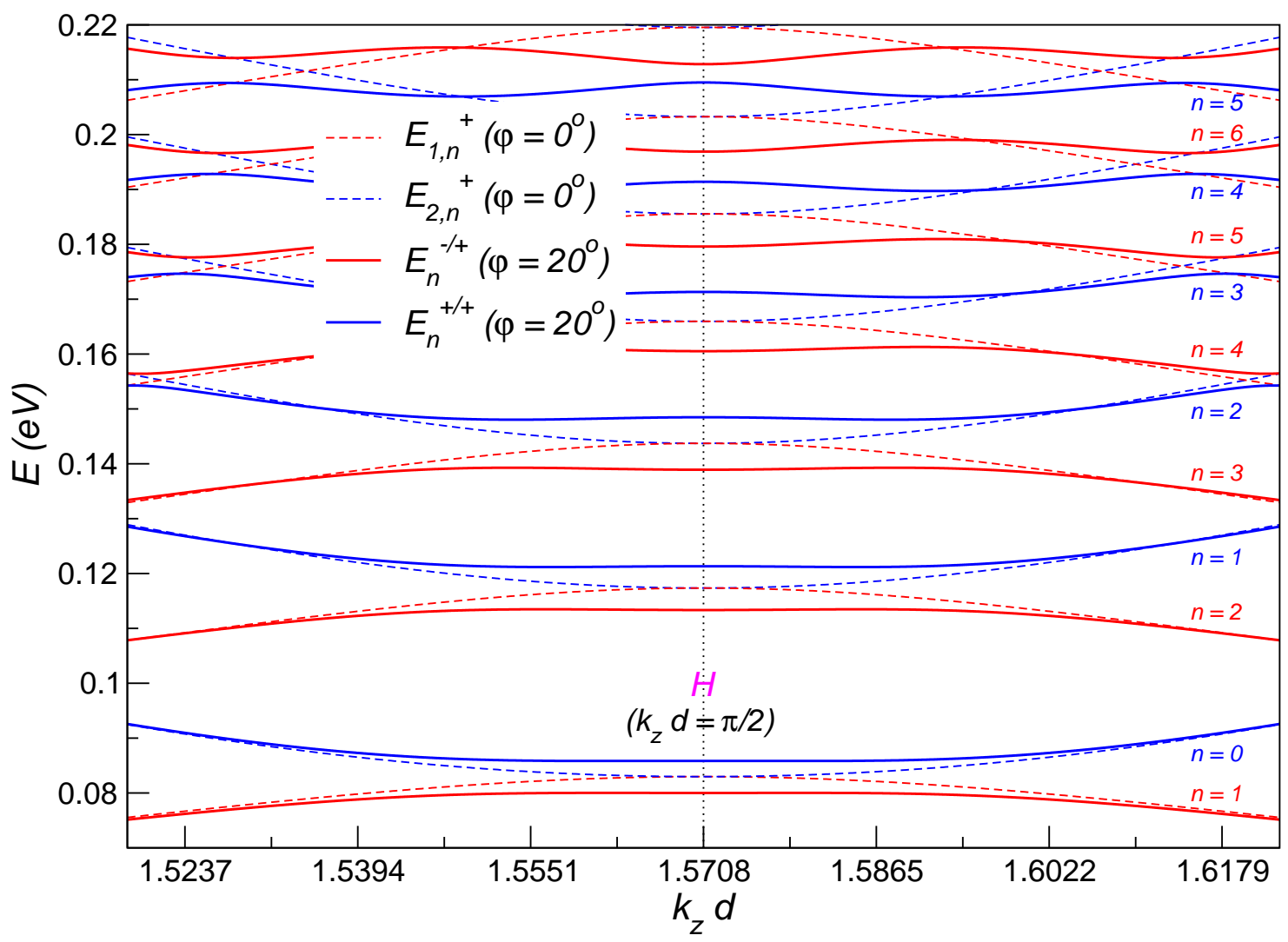

Figure 6. (Color online) Graphite Landau subbands, depicted by the rectangular box in Fig. 2, as a function of $k_{z}$ nearby the $H$ point. Dashed curves are graphite LLs, $E_{1, n}^{+}$and $E_{2, n}^{+}$, in the perpendicular magnetic field $B_{z}=5 \mathrm{~T}, \varphi=0^{\circ}$. Solid curves are LLs of graphite, $E_{n}^{-/+}$and $E_{n}^{+/+}$, subject to the tilted magnetic field with the perpendicular component $B_{z}=5 \mathrm{~T}$ and the tilt angle $\varphi=20^{\circ}$. 
orientation, $B_{y} \gg B_{z}$, as $\eta_{d} \rightarrow \infty$ for $B_{z} \rightarrow 0$, and the expansion in powers of $\eta_{d}$ is no longer acceptable.

Also the perturbation theory is less appropriate for states with large $n$, as the energy difference between neighboring LLs is smaller then for states with small $n$ and,

moreover, the interlayer coupling matrix elements, $\widetilde{\mathcal{T}}_{n, n \pm 1}$, increase with $\sqrt{n}$. The limits of the numerical approach are not clear at present, but we should have in mind that from the experimental point of view the angles with almost in-plane orientation are not so interesting due to the mosaic structure of most graphite crystals.

\section{Conclusion}

Based on the simple nearest-neighbor tight-binding quantum mechanical model, we presented the calculation method of the band structure of Bernal-stacked graphite subject to tilted magnetic fields. We applied the lowest order perturbation theory to obtain analytic solutions of the formulated equations, the accuracy of which was later checked by the simplified numerical calculation. The special attention has been paid to the field dependence of the LLs at the $K$ and $H$ points of the graphite Brillouin zone where the density of states exhibits van Hove singularities in the perpendicular magnetic field. We have found that at the $K$ point, where the electron structure in the perpendicular magnetic field reminds strongly that of the bilayer graphene, the influence of the in-plane component of the magnetic field is negligible. On the other hand, at the $H$ point, where the electron structure mimics the behavior of the Dirac fermions, the application of the tilted magnetic field leads to the splitting of LLs. This splitting is of the order of several meV, which is an experimentally observable value, and it grows with increasing of the in-plane component of the magnetic field.

\section{Acknowledgements}

The authors benefited from discussions with Milan Orlita. The support of the European Science Foundation EPIGRAT project (GRA/10/E006), AV CR research program AVOZ10100521 and the Ministry of Education of the Czech Republic project LC510 is acknowledged.

[1] J. W. McClure, Phys. Rev. 104, 666 (1956).

[2] J. W. McClure, Phys. Rev. 119, 606 (1960).

[3] M. Inoue, J. Phys. Soc. Jpn. 17, 808 (1962).

[4] O. P. Gupta and P. R. Wallace, Phys. Status Solidi B 54, 53 (1972).

[5] G. Dresselhaus, Phys. Rev. B 10, 3602 (1974).

[6] K. Nakao, J. Phys. Soc. Jpn. 40, 761 (1976).

[7] L. Onsager, Philos. Mag. 43, 1006 (1952).

[8] E. M. Lifshitz, Sov. Phys. - JETP 30, 63 (1956).

[9] L. L. Chang, E. E. Mendez, N. J. Kawai, L. Esaki, Surface Sci. 113, 306 (1982).

[10] H. L. Stormer, J. P. Eisenstein, A. C. Gossard, W. Wiegmann, K. Baldwin, Phys. Rev. Lett. 56, 85 (1986). 
[11] O. Jaschinski, G. Nachtwei, J. Schoenes, P. Bönsch, A. Schlachetzki, Physica B 251, 873 (1998).

[12] G. Nachtwei, O. Jaschinski, H. Künzel, J. Appl. Phys. 84, 323 (1998).

[13] M. Kawamura, A. Endo, S. Katsumoto, Y. Iye, C. Terakura, S. Uji, Physica B 298, 48 (2001).

[14] N. A. Goncharuk, L. Smrčka, P. Svoboda, P. Vašek, J. Kučera, Yu. Krupko, W. Wegscheider, Phys. Rev. B 75, 245322 (2007).

[15] R. Dingle, Surf. Sci. 73, 229 (1978).

[16] M. V. Kartsovnik, P. A. Kononovich, V. N. Laukhin, I. F. Shchegolev, JETP Lett. 48, 541 (1988).

[17] K. Kajita, Y. Nishio, T. Takahashi, W. Sasaki, R. Kato, H. Kobayashi, Sol. St. Commun. 70, 1129 (1989).

[18] K. Yamaji, J. Phys. Soc. Jpn. 58, 1520 (1989).

[19] R. Yagi, Y. Iye, T. Osada, S. Kagoshima, J. Phys. Soc. Jpn. 59, 3069 (1989).

[20] A. G. Lebed, Per Bak, Phys. Rev. Lett. 63, 1315 (1989).

[21] T. Osada and A. Kawasumi and S. Kagoshima and N. Miura and G. Saito, Phys. Rev. Lett. 66, 1525 (1991).

[22] G.M. Danner, W. Kang, P.M. Chaikin, Phys. Rev. Lett. 72, 3714 (1994).

[23] E. I. Chashechkina and P. M. Chaikin, Phys. Rev. Lett. 80, 2181 (1998).

[24] I. J. Lee, M. J. Naughton, Phys. Rev. B 57, 7423 (1998).

[25] Y. Iye, M. Baxendale, V. Z. Mordkovich, J. Phys. Soc. Jpn. 63, 1643 (1994).

[26] K. Enomoto, S. Uji, T. Yamaguchi, T. Terashima, T. Konoike, M. Nishimura, T. Enoki, M. Suzuki, I. S. Suzuki, Phys. Rev. B 73, 045115 (2006).

[27] S. S. Pershoguba, V. M. Yakovenko, Phys. Rev. B 82, 205408 (2010).

[28] Y.-H. Hyun, Y. Kim, C. Sochichiu, M.-Y. Choi, arXiv:1008.0488v1 (2010).

[29] M. Koshino, T. Ando, Phys. Rev. B 77, 115313 (2008).

[30] M. Orlita, C. Faugeras, J. M. Schneider, G. Martinez, D. K. Maude, M. Potemski, Phys. Rev. Lett. 102, 166401 (2009).

[31] E. A. Henriksen, Z. Jiang, L.-C. Tung, M. E. Schwartz, M. Takita, Y.-J. Wang, P. Kim, H. L. Stormer, Phys. Rev. Lett. 100, 087403 (2008).

[32] K.-C. Chuang, A. M. R. Baker, R. J. Nicholas, Phys. Rev. B 80, 161410 (2009).

[33] M. Orlita, M. Potemski, Semicond. Sci. Technol. 25, 063001 (2010).

[34] N. Ubrig, P. Plochocka, P. Kossacki, M. Orlita, D. K. Maude, O. Portugall, G. L. J. A. Rikken, Phys. Rev. B 83, 073401 (2011).

[35] N. A. Goncharuk, L. Smrčka, J. Kučera, K. Výborný, Phys. Rev. B 71, 195318 (2005).

[36] I. S. Gradshtejn, I. M. Ryzhik, Tables of integrals, sums, rows and products, Moscow (1963).

[37] D. I. Blokhincev, Foundations of Quantum Mechanics, Nauka, Moscow, 1976 (in Russian). 